Pacific Journal of Mathematics

ORDERED COMPLETELY REGULAR SEMIGROUPS 


\section{ORDERED COMPLETELY REGULAR SEMIGROUPS}

\section{Tôru SaITô}

Introduction. In the previous papers [7], [8], we studied some special types of ordered semigroups in our general sense (cf. $\S 1$ ). In the continuation of our study, in this note we characterize ordered completely regular semigroups.

In the algebraic theory completely regular semigroups were first studied systematically by Clifford [1]. He characterized these semigroups when the idempotents of the semigroup commute. Recently Fantham [4] generalized this result and characterized completely regular semigroups when the idempotents of the semigroup constitute a subsemigroup.

Ordered completely regular semigroups are shown, in this note, to be semigroups, in which the idempotents constitute subsemigroups. However, it can be shown that the abstract semigroup of an ordered completely regular semigroup is simpler than the semigroups of Fantham's type. Indeed, our main theorem (Theorem 6) asserts that an ordered completely regular semigroup is characterized by the ordered idempotent semigroup constituted by all the idempotents of the semigroup, the ordered groups corresponding to all the elements of the associated semilattice, and the mappings between these ordered groups corresponding to all comparable pairs of elements of the associated semilattice which satisfy certain conditions.

We remark, we characterize, in $\S 3$, ordered completely simple semigroups without zero. This characterization seems to be interesting by itself, by virtue of the importance of completely simple semigroups without zero.

1. Preliminaries. A semigroup $S$ is called completely regular, if, for every element $a \in S$, there exists $x \in S$ such that

$$
a x a=a \text { and } a x=x a
$$

(Lyapin [5]). Clifford called such a semigroup a semigroup admitting relative inverses and gave many interesting results of this semigroup. Here we mention some of them without proofs.

LEMma 1 (Clifford [1], Theorem 1). A semigroup is completely regular if and only if it is the set-union of mutually disjoint groups.

The adjective 'mutually disjoint' in the above Lemma 1 can be

Received March 27, 1963. 
omitted (cf. Clifford [2], p. 501).

Lemma 2 (Clifford [2], Theorem 6). A semigroup is completely regular if and only if it is a semilattice of completely simple semigroups without zero.

Thus, a completely regular semigroup $S$ determines a semilattice $T_{S}$ such that to each element $\alpha$ of $T_{s}$ there corresponds a subsemigroup $S(\alpha)$ of $S$ with the following properties:

(a) the $S(\alpha)$ are mutually disjoint and their union is $S$;

(b) each $S(\alpha)$ is a completely simple semigroup without zero;

(c) $S(\alpha) S(\beta) \subseteq S(\alpha \circ \beta)$, where $\alpha \circ \beta$ is the product of $\alpha$ and $\beta$ in $T_{s}$.

We call the semilattice $T_{s}$ the associated semilattice of the completely regular semigroup $S$.

For discussing the structure of semigroups, it is sometimes successful to use ideal-theoretical notions. In this note, we use $R$-equivalence, L-equivalence and D-equivalence in the sense of Miller and Clifford [6]. It can be seen that the decomposition of a completely regular semigroup $S$ into the collection of subsemigroups $S(\alpha)$ mentioned in Lemma 2, coincides with the decomposition of $S$ into the collection of $D$-equivalence classes.

In particular, a completely simple semigroup without zero is completely regular.

When a completely simple semigroup without zero is left simple, that is, it consists of only one $L$-equivalence class, it is called a left group. A right group is defined similarly. A semigroup $I$ is called left singular, if the multiplication is defined by

$$
a b=a \text { for every } a, b \in I .
$$

A left singular semigroup is clearly a left group. A right singular semigroup is defined similarly. The following lemma is well-known (cf. Cohn [3], p. 172).

LEMMA 3. A left group is semigroup-isomorphic to the direct product of a group and a left singular semigroup. A right group is semigroup-isomorphic to the direct product of a group and a right singular semigroup.

By an ordered semigroup, we mean a semigroup $S$ with?a simple order $<$ satisfying the following condition:

$$
a \leqq b \quad \text { implies } a c \leqq b c \text { and } c a \leqq c b \text {. }
$$


Evidently a left or right singular semigroup turns out to be an ordered semigroup if we order the elements of the semigroup arbitrarily.

Ordered semigroups were studied in our previous papers [7], [8]. Here we mention some elementary results from these papers without proofs.

Lemma 4 (Saitô [8], Corollary of Lemma 1). The set of all idempotents of an ordered semigroup $S$, if it is non-void, is a subsemigroup of $S$.

Lemma 5 (Saitô [7], Lemma 2). If $a$ and $b$ are elements of an ordered idempotent semigroup $E$ such that $a \leqq b$, then

$$
a \leqq a b \leqq b \quad \text { and } \quad a \leqq b a \leqq b .
$$

Lemma 6 (Saitô [7], Theorem 1). In an ordered idempotent semigroup $E$, each $D$-equivalence class consists of either onlg one $L$ equivalence class or only one R-equivalence class.

In an ordered idempotent semigroup $E$, a $D$-equivalence class is called $L$-typed, if it consists of only one $L$-equivalence class, while it is called $R$-typed, if it consists of only one $R$-equivalence class.

2. In this section, we always denote by $S$ an ordered completely regular semigroup, and by $E$ the set of all the idempotents of $S$. $E$ is nonvoid, and so, by Lemma 4, constitutes a subsemigroup of $S$. The ordered semigroup $E$ is evidently completely regular, and so $E$ is decomposed into the collection of mutually disjoint subsemigroups $E(\delta)$ of $E$ in such a way as is described in Lemma 2.

Lemma 7. For $f, f^{\prime} \in E$,

(a) $f \equiv f^{\prime}(L)$ in $S$ if and only if $f \equiv f^{\prime}(L)$ in $E$;

(b) $f \equiv f^{\prime}(R)$ in $S$ if and only if $f \equiv f^{\prime}(R)$ in $E$;

(c) $f \equiv f^{\prime}(D)$ in $S$ if and only if $f \equiv f^{\prime}(D)$ in $E$.

Proof. (a) If $f \equiv f^{\prime}(L)$ in $S$, then there exist elements $x, y \in S$ such that $x f=f^{\prime}, y f^{\prime}=f$. Then we have $f^{\prime} f=f^{\prime}, f f^{\prime}=f$, and so $f \equiv f^{\prime}(L)$ in $E$. It is trivial that $f \equiv f^{\prime}(L)$ in $E$ implies $f \equiv f^{\prime}(L)$ in $S$. (b) The proof is similar as in (a). (c) If $f \equiv f^{\prime}(D)$ in $S$, then there exists an element $z \in S$ such that $f \equiv z(L), z \equiv f^{\prime}(R)$ in $S$. By Lemma 1 , there exists a subgroup which contains $z$, and let $e$ be the identity of this subgroup. Then clearly $e \in E$ and $z \equiv e(L), z \equiv e(R)$ in $S$. Hence $f \equiv e(L), e \equiv f^{\prime}(R)$ in $S$, and so, taking account of (a) and (b) proved above, we have $f \equiv f^{\prime}(D)$ in $E$. It is trivial that $f \equiv f^{\prime}(D)$ in $E$ implies $f \equiv f^{\prime}(D)$ in $S$. 
Let $\left\{S(\alpha) ; \alpha \in T_{S}\right\}$ be the decomposition of $S$ described in Lemma 2. Clearly each $D$-equivalence class $S(\alpha)$ in $S$ contains with every $a \in S(\alpha)$ the identity of a subgroup which contains $a$. Hence $S(\alpha) \cap E \neq \phi$ for every $\alpha \in T_{s}$. Moreover, by Lemma $7(c), S(\alpha) \cap E$ is a member of the decomposition $\left\{E(\delta) ; \delta \in T_{E}\right\}$ of $E$. Therefore it can be seen that the associated semilattice $T_{S}$ of $S$ is semilattice-isomorphic to the associated semilattice $T_{E}$ of $E$. Thus in what follows, without loss of generality, we assume that the semilattice $T_{E}$ coincides with the semilattice $T_{s}$.

Now we give a definition. A semilattice $T$ is called a tree semilattice if it satisfies the following condition:

if $\alpha, \alpha^{\prime}, \beta, \beta^{\prime}$ are elements of $T$ such that $\alpha \leqq \alpha^{\prime}, \beta \leqq \beta^{\prime}$ and $\alpha$ and $\beta$ are non-comparable, then $\alpha^{\prime}$ and $\beta^{\prime}$ are non-comparable. (Here and hereafter we denote by $\prec$ the order in a semilattice. $a \leqq \beta$ in a semilattice is defined to mean $\alpha \circ \beta=\alpha$.)

Lemma 8 (Saitô [7], Theorem 3). The associated semilattice of an ordered idempotent semigroup is a tree semilattice.

As an immediate corollary of the above Lemma 8, we obtain

THEOREM 1. The associated semilattice $T_{s}$ of an ordered completely regular semigroup $S$ is a tree semilattice.

3. In this section, we characterize ordered completely simple semigroups without zero.

For brevity, in this section we denote by $S$ an ordered completely simple semigroup without zero. By Lemma 7 , the ordered idempotent semigroup $E$ which consists of all the idempotents of $S$, constitutes a $D$-equivalence class in $E$. Hence, by Lemma $6, E$ itself constitutes either an $L$-equivalence class or an $R$-equivalence class in $E$. In the former case, the ordered completely simple semigroup $S$ without zero is called $L$-typed, while in the latter case $S$ is called $R$-typed. In the rest of this section, we treat only the $L$-typed case. The $R$-typed case can be discussed similarly.

Lemma 9. In this L-typed case, an ordered completely simple semigroup $S$ without zero is a left group.

Proof. Let $a, b \in S$, and let $e$ and $e^{\prime}$ be the identities of subgroups containing $a$ and $b$, respectively. Then

$$
a \equiv e \equiv e^{\prime} \equiv b(L),
$$

and so $S$ is left simple. 
Since $S$ is a left group, it is, by Lemma 3, semigroup-isomorphic to the direct product of a group $G$ and a left singular semigroup $I$. Thus we may consider that $S$ consists of elements $(g, i)$ with $g \in G$, $i \in I$, and the multiplication in $S$ is ruled by

$$
(g, i)\left(g^{\prime}, i^{\prime}\right)=\left(g g^{\prime}, i\right) \text {. }
$$

We denote the identity of the group $G$ by $e$.

New we show that

$$
(g, i)<\left(g^{\prime}, i\right) \text { if and only if }\left(g, i^{\prime}\right)<\left(g^{\prime}, i^{\prime}\right) .
$$

In fact, if $(g, i)<\left(g^{\prime}, i\right)$, then

$$
(e, i)\left(g, i^{\prime}\right)=(g, i)<\left(g^{\prime}, i\right)=(e, i)\left(g^{\prime}, i^{\prime}\right),
$$

and so $\left(g, i^{\prime}\right)<\left(g^{\prime}, i^{\prime}\right)$.

Thus the order in $G$ is well-defined, if we define

$$
g<g^{\prime} \text { in } G \text { if and only if }(g, i)<\left(g^{\prime}, i\right) \text {. }
$$

Then, as is easily verified, $G$ is an ordered group or, more exactly, a simply ordered group.

Moreover, we define the order in $I$ by

$$
i<i^{\prime} \text { in } I \text { if and only if }(e, i)<\left(e, i^{\prime}\right) \text {. }
$$

Then clearly $I$ is an ordered left singular semigroup.

Now we show that

$$
\begin{aligned}
& (g, i)<\left(g^{\prime}, i^{\prime}\right) \text { if and only if either } g<g^{\prime} \\
& \text { or } g=g^{\prime}, i<i^{\prime} \text {. }
\end{aligned}
$$

In fact, suppose that $(g, i)<\left(g^{\prime}, i^{\prime}\right)$. If $g \neq g^{\prime}$, then

$$
(g, i)=(e, i)(g, i) \leqq(e, i)\left(g^{\prime}, i^{\prime}\right)=\left(g^{\prime}, i\right),
$$

and so $g<g^{\prime}$. If $g=g^{\prime}$, then

$$
(e, i)=(g, i)\left(g^{-1}, i\right) \leqq\left(g^{\prime}, i^{\prime}\right)\left(g^{-1}, i\right)=\left(e, i^{\prime}\right) .
$$

But, in this case, $i \neq i^{\prime}$, and so $i<i^{\prime}$. Conversely, if $g<g^{\prime}$, then

$$
(e, i)(g, i)=(g, i)<\left(g^{\prime}, i\right)=(e, i)\left(g^{\prime}, i^{\prime}\right),
$$

and so $(g, i)<\left(g^{\prime}, i^{\prime}\right)$. If $g=g^{\prime}, i<i^{\prime}$, then

$$
(g, i)\left(g^{-1}, i\right)=(e, i)<\left(e, i^{\prime}\right)=\left(g^{\prime}, i^{\prime}\right)\left(g^{-1}, i\right),
$$

and so $(g, i)<\left(g^{\prime}, i^{\prime}\right)$.

Now we remark that an element of $S$ is idempotent if and only 
if it has the form $(e, i)$ with $i \in I$. Moreover it is easily verified that the mapping

$$
E \ni(e, i) \rightarrow i \in I
$$

is an ordered-semigroup-isomorphism of $E$ onto $I$. Thus, we may consider that the ordered left singular semigroup $I$ coincides with the ordered semigroup $E$. By this convention, the idempotent $f$ of $S$ is identified with $(e, f)$.

THEOREM 2. An ordered completely simple semigroup $S$ without zero is isomorphic as ordered semigroup to the lexicographically ordered direct product of an ordered group $G$ and the ordered left or right singular semigroup $E$ which is constituted by all the idempotents of $S$. Conversely an ordered semigroup which is isomorphic to the lexicographically ordered direct product of an ordered group and an ordered left or right singular semigroup is an ordered completely simple semigroup without zero.

The proof of the converse part of Theorem 2 can be verified easily.

4. In this section we discuss the structure of ordered completely regular semigroups. First of all, we mention some results from our previous paper [7].

Lemma 10 (Saitô [7], Lemma 4). Let $f, f^{\prime}, f^{\prime \prime}$ be elements of an ordered idempotent semigroup $E$ such that $f \leqq f^{\prime \prime} \leqq f^{\prime}$, and let $E(\alpha)$, $E(\beta), E(\gamma)$ be the $D$-equivalence classes which contain $f, f^{\prime}, f^{\prime \prime}$, respectively. Then $\gamma \geqq \alpha \circ \beta$ in the semilattice $T_{E}$.

Lemma 11 (Saitô [7], Theorem 2). Let $f$ and $f^{\prime}$ be elements of an ordered idempotent semigroup $E$ such that $f \leqq f^{\prime}$, and let $f \in E(\alpha)$, $f^{\prime} \in E(\beta)$. If the $D$-equivalence class $E(\alpha \circ \beta)$ is L-typed, then

$$
\begin{aligned}
& f f^{\prime}=\min \{y ; y \in E(\alpha \circ \beta) \text { and } y \geqq f\}, \\
& f^{\prime} f=\max \left\{y ; y \in E(\alpha \circ \beta) \text { and } y \leqq f^{\prime}\right\} .
\end{aligned}
$$

If $E(\alpha \circ \beta)$ is $R$-typed, then

$$
\begin{aligned}
& f f^{\prime}=\max \left\{y ; y \in E(\alpha \circ \beta) \text { and } y \leqq f^{\prime}\right\}, \\
& f^{\prime} f=\min \{y ; y \in E(\alpha \circ \beta) \text { and } y \geqq f\} .
\end{aligned}
$$

Now we show the following

LEMMA 12. Let $a$ be an element of an ordered completely regular semigroup $S$, let $f$ be the identity element of a group which contains 
$a$, and let $f^{\prime}$ be an arbitrary idempotent of $S$. Then a and $f f^{\prime} f$ are permutable.

Proof. Let $S(\alpha)$ and $S(\beta)$ be the $D$-equivalence classes which contain $f$ and $f^{\prime}$, respectively. We set $f^{\prime \prime}=a f f^{\prime} f a^{-1}$, where we denote by $a^{-1}$ the inverse element of $a$ in a group which contains $a$. Then $a, a^{-1} \in S(\alpha)$ and $f^{\prime \prime} \in S(\alpha \circ \beta)$. Moreover, as is easily seen, $f^{\prime \prime}$ is idempotent and $f f^{\prime \prime} f=f^{\prime \prime}$. Now we suppose that $f \leqq f^{\prime}$ and that, in the ordered idempotent semigroup $E$ which consists of all the idempotents of $S, E(\alpha \circ \beta)$ is an $L$-typed $D$-equivalence class. Then, by Lemma $5, f \leqq f f^{\prime} f$, and so

$$
f=a f a^{-1} \leqq a\left(f f^{\prime} f\right) a^{-1}=f^{\prime \prime}, \quad f \leqq f^{\prime \prime} f .
$$

Hence, by Lemma 11,

$$
f^{\prime \prime}=f f^{\prime \prime} f=\min \{y ; y \in E(\alpha \circ \beta) \text { and } y \geqq f\}=f f^{\prime} f .
$$

Similarly we can prove $f^{\prime \prime}=f f^{\prime} f$ in the remaining cases. The equality $a\left(f f^{\prime} f\right) a^{-1}=f^{\prime \prime}=f f^{\prime} f$ implies evidently that $a$ and $f f^{\prime} f$ are permutable.

Each $D$-equivalence class $S(\alpha)$ of an ordered completely regular semigroup $S$ is, by Lemma 2, a completely simple semigroup without zero. Hence, by Theorem 2, we may consider, without loss of generality, $S(\alpha)$ is the lexicographically ordered direct product of an ordered group $G(\alpha)$ and the ordered left or right singular semigroup $E(\alpha)$ constituted by all the idempotents of $S(\alpha)$.

The groups $G(\alpha)\left(\alpha \in T_{s}\right)$ are called the structure groups of the ordered completely regular semigroup $S$. The identity of the group $G(\alpha)$ is denoted by $e_{\alpha}$. If an element $a$ of $S(\alpha)$ is represented by $(g, f)$ with $g \in G(\alpha), f \in E(\alpha)$, then $g$ is called the group component of $a$ and $f$ is called the singular component of $a$.

LEMMA 13. If $a \in S(\alpha), f \in E(\alpha)$, then both af and fa have the same group component as that of $a$. Conversely, if $a, a^{\prime} \in S(\alpha)$, and if $a$ and $a^{\prime}$ have the same group component, then there exists $f \in E(\alpha)$ such that $a^{\prime}=$ faf.

Proof. Since $f \in E(\alpha)$ has the group component $e_{\alpha}$, the first half of this lemma is clear. For the second half, we assume $a=\left(g, f^{\prime}\right)$ and $a^{\prime}=(g, f)$. Then, as is easily seen, the element $f=\left(e_{\alpha}, f\right) \in E(\alpha)$ satisfies the required equality $\alpha^{\prime}=f a f$.

Let $\alpha$ and $\beta$ be elements of the associated semilattice $T_{s}$ such that $\alpha \geqq \beta$, and $g$ be an element of the structure group $G(\alpha)$. We take an element $a \in S(\alpha)$ with the group component $g$, and also take 
an idempotent $f \in E(\beta)$. Then we consider the group component $g^{\prime}$ of the element $f a f$. Since $f a f \in S(\beta)$, we have $g^{\prime} \in G(\beta)$. Now we show the following

Lemma 14. The element $g^{\prime}$ of $G(\beta)$ constructed above is determined only by $g \in G(\alpha)$, irrespective of the choice of $a \in S(\alpha)$ and $f \in E(\beta)$.

Proof. Let $\alpha^{\prime}$ be an element of $S(\alpha)$ with the group component $g$ and let $f^{\prime}$ be an element of $E(\beta)$. Then, by Lemma 13, there exists an element $h \in E(\alpha)$ such that $\alpha^{\prime}=h a h$. First we assume that the $D$-equivalence class $E(\beta)$ of the ordered idempotent semigroup $E$ is $L$-typed. Then $f^{\prime} h f=f^{\prime}=f^{\prime} h$ and so

$$
f^{\prime} a^{\prime} f^{\prime}=f^{\prime} h a h f^{\prime}=f^{\prime} h f a h f^{\prime} .
$$

Using Lemma 13 repeatedly, $f a f$ and $f a$ have the same group component, and so $f a f$ and $f^{\prime} a^{\prime} f^{\prime}=f^{\prime} h(f a) h f^{\prime}$ have the same group component. In the case when $E(\beta)$ is $R$-typed, we can prove the assertion of the lemma in a similar way.

Now, for $\alpha, \beta \in T_{S}$ such that $\alpha \geqq \beta$, we define the mapping $\varphi_{\beta}^{\alpha}$ of the structure group $G(\alpha)$ into the structure group $G(\beta)$ by

$$
\varphi_{\beta}^{\alpha}: G(\alpha) \ni g \rightarrow g^{\prime} \in G(\beta),
$$

where $g^{\prime}$ is the element of $G(\beta)$ described above. By Lemma 14, $g^{\prime}$ is well-defined by $g \in G(\alpha)$.

The mappings $\varphi_{\beta}^{\alpha}$ defined for $\alpha, \beta \in T_{S}$ such that $\alpha \geqq \beta$, are called the structure mappings of the ordered completely regular semigroup $S$.

THEOREM 3. The structure mappings $\varphi_{\beta}^{\alpha}$ of an ordered completely regular semigroup $S$ have the following properties:

(a) $\varphi_{\alpha}^{\alpha}$ is the identity mapping of $G(\alpha)$;

(b) if $\alpha \geqq \beta \geqq \gamma$, then $\varphi_{\beta}^{\alpha} \varphi_{\gamma}^{\beta}=\varphi_{\gamma}^{\alpha}$;

(c) $\varphi_{\beta}^{\alpha}$ is a group-homomorphism of $G(\alpha)$ into $G(\beta)$;

(d) $\varphi_{\beta}^{\alpha}$ is isotone:

for $g, g^{\prime} \in G(\alpha)$ with $g \leqq g^{\prime}$, we have $g \varphi_{\beta}^{\alpha} \leqq g^{\prime} \varphi_{\beta}^{\alpha}$ in $G(\beta)$. (We remark, in this note we denote by $g \varphi$ the image of an element $g$ by a mapping $\varphi$, and denote by $\varphi \psi$ the mapping which maps $g$ into $\left.(g \varphi) \psi_{.}\right)$

Proof. (a) Evident by Lemma 13. (b) Suppose that $g \varphi_{\beta}^{\alpha}=g^{\prime}$, $g^{\prime} \varphi_{\gamma}^{\beta}=g^{\prime \prime}$, and that $f \in E(\beta), h \in E(\gamma)$. For an element $a$ with the group component $g$, by Lemma 13, af has the group component $g^{\prime}$, and so $a f h$ has the group component $g^{\prime \prime}$. Since $f h \in E(\gamma), g \varphi_{\gamma}^{\alpha}$ is the 
group conponent of $a f h$ and so equals to $g^{\prime \prime}$. (c) Let $g, g^{\prime} \in G(\alpha)$, and let $a$ and $a^{\prime}$ be elements of $S(\alpha)$ with the group components $g$ and $g^{\prime}$, respectively. By Lemma 12 , there exists $f \in E(\beta)$ which is permutable with $a^{\prime}$. Hence $a a^{\prime} f=(a f)\left(a^{\prime} f\right)$. The element $a a^{\prime}$ is an element of $S(\alpha)$ and has the group component $g g^{\prime}$, and so, by comparing the group components on both sides of the above equality, we have

$$
g g^{\prime} \varphi_{\beta}^{\alpha}=\left(g \varphi_{\beta}^{\alpha}\right)\left(g^{\prime} \varphi_{\beta}^{\alpha}\right) \text {. }
$$

(d) Suppose that $g \leqq g^{\prime}$ in $G(\alpha)$ and let $a$ and $\alpha^{\prime}$ be elements of $S(\alpha)$ with the group components $g$ and $g^{\prime}$ respectively but with the same singular component. Then, by (2) in $\S 3$, we have $a \leqq a^{\prime}$, and so $a f \leqq a^{\prime} f$ for $f \in E(\beta)$. Now $a f$ and $a^{\prime} f$ belong to the same $D$-equivalence class $S(\beta)$. Hence, comparing the group components on both sides of the above inequality, we have, by (4) in $\S 3$,

$$
g \varphi_{\beta}^{\alpha} \leqq g^{\prime} \varphi_{\beta}^{\alpha} .
$$

THEOREM 4. For $(g, f) \in S(\alpha)$ and $\left(g^{\prime}, f^{\prime}\right) \in S(\beta)$,

$$
(g, f)\left(g^{\prime}, f\right)=\left(\left(g \varphi_{\alpha_{\circ} \beta}^{\alpha}\right)\left(g^{\prime} \varphi_{\alpha \circ \beta}^{\beta}\right), f f^{\prime}\right) \text {. }
$$

Proof. First we prove

$$
(g, f)\left(f f^{\prime} f\right)=\left(f f^{\prime} f\right)(g, f)=\left(g \varphi_{\alpha \circ \beta}^{\alpha}, f f^{\prime} f\right) .
$$

In fact, $f=\left(e_{\alpha}, f\right)$ is easily seen to be the identity of a group which contains the element $(g, f)$. Hence, by Lemma 12,

$$
(g, f)\left(f f^{\prime} f\right)=\left(f f^{\prime} f\right)(g, f)\left(f f^{\prime} f\right)=\left(f f^{\prime} f\right)(g, f) \text {. }
$$

Moreover $f f^{\prime} f \in E(\alpha \circ \beta)$, and so the group component of $\left(f f^{\prime} f\right)(g, f)\left(f f^{\prime} f\right)$ is $g \varphi_{\alpha \circ \beta}^{\alpha}$. Furthermore, since $\left(f f^{\prime} f\right)(g, f)\left(f f^{\prime} f\right) \in S(\alpha \circ \beta)$, the element

$$
\left(f f^{\prime} f\right)(g, f)\left(f f^{\prime} f\right)=\left(f f^{\prime} f\right)\left(\left(f f^{\prime} f\right)(g, f)\left(f f^{\prime} f\right)\right)\left(f f^{\prime} f\right)
$$

has the same singular component as that of $f f^{\prime} f=\left(e_{\alpha_{\circ} \beta}, f f^{\prime} f\right)$. Hence we have the relation (6).

Now we have

$$
\begin{aligned}
(g, f) & \left(g^{\prime}, f^{\prime}\right)=(g, f) f f^{\prime}\left(g^{\prime}, f^{\prime}\right) \\
& =(g, f)\left(f f^{\prime} f\right)\left(f^{\prime} f f^{\prime}\right)\left(g^{\prime}, f^{\prime}\right) \quad \text { since } f f^{\prime} \text { is idempotent, } \\
& =\left(g \varphi_{\alpha_{\circ \beta}}^{\alpha}, f f^{\prime} f\right)\left(g^{\prime} \varphi_{\alpha_{\circ \beta}}^{\beta}, f^{\prime} f f^{\prime}\right) \quad \text { by }(6), \\
& =\left(\left(g \varphi_{\alpha_{\beta} \beta}^{\alpha}\right)\left(g^{\prime} \varphi_{\alpha \circ \beta}^{\beta}\right), f f^{\prime} f f^{\prime} f f^{\prime}\right) \text { by the multiplication in } S(\alpha \circ \beta), \\
& =\left(\left(g \varphi_{\alpha_{\circ} \beta}^{\alpha}\right)\left(g^{\prime} \varphi_{\alpha_{\circ} \beta}^{\beta}\right), f f^{\prime}\right) .
\end{aligned}
$$

LEMmA 15. Let $f \in E(\alpha), f^{\prime} \in E(\beta), \alpha \geqq \beta$ and $g \in G(\alpha)$. 
(a) If $f \leqq f^{\prime}$ in $S$, then $(g, f) \leqq\left(g \varphi_{\beta}^{\alpha}, f^{\prime}\right)$.

(b) If $f \geqq f^{\prime}$ in $S$, then $(g, f) \geqq\left(g \varphi_{\beta}^{x}, f^{\prime}\right)$.

Proof. We prove only (a) in the case when $E(\beta)$ is $L$-typed. Then, by Lemma 11, $f^{\prime} f=f^{\prime}$. Since

$$
\left(e_{\alpha}, f\right)=f \leqq f^{\prime}=\left(e_{\beta}, f^{\prime}\right),
$$

we have, by Theorem 4 ,

$$
(g, f)=\left(e_{\alpha}, f\right)(g, f) \leqq\left(e_{\beta}, f^{\prime}\right)(g, f)=\left(g \varphi_{\beta}^{\alpha}, f^{\prime}\right) .
$$

THEOREM 5. For $(g, f) \in S(\alpha)$, and $\left(g^{\prime}, f^{\prime}\right) \in S(\beta),(g, f)<\left(g^{\prime}, f^{\prime}\right)$ if and only if either $g \varphi_{\alpha \circ \beta}^{\alpha}<g^{\prime} \varphi_{\alpha \circ \beta}^{\beta}$ or $g \varphi_{\alpha \circ \beta}^{\alpha}=g^{\prime} \varphi_{\alpha \circ \beta}^{\beta}, f<f^{\prime}$.

Proof. Without loss of generality, we assume that $E(\alpha \circ \beta)$ is $L$-typed. Then $f f^{\prime} f=f f^{\prime}=f f^{\prime} f^{\prime}$. Suppose that

$$
g \varphi_{\alpha \circ \beta}^{\alpha} \geqq g^{\prime} \varphi_{\alpha \circ \beta}^{\beta} .
$$

Then we have

$$
\begin{aligned}
\left(g \varphi_{\alpha \circ \beta}^{\alpha}, f f^{\prime}\right) & =\left(e_{\alpha \circ \beta}, f f^{\prime}\right)(g, f) \\
& \leqq\left(e_{\alpha \circ \beta}, f f^{\prime}\right)\left(g^{\prime}, f^{\prime}\right)=\left(g^{\prime} \varphi_{\alpha^{\circ} \beta}^{\beta}, f f^{\prime}\right),
\end{aligned}
$$

and so $g \varphi_{\alpha \circ \beta}^{\alpha} \leqq g^{\prime} \varphi_{\alpha \circ \beta}^{\beta}$. Hence $g \varphi_{\alpha \circ \beta}^{\alpha}=g^{\prime} \varphi_{\alpha \circ \beta}^{\beta}$. Now we suppose that $f \geqq f^{\prime}$ were true. Then, by Lemma $5, f \geqq f f^{\prime} \geqq f$. Hence, by Lemma 15 , we would have

$$
(g, f) \geqq\left(g \varphi_{a \circ \beta}^{\alpha}, f f^{\prime}\right)=\left(g^{\prime} \varphi_{\alpha_{\circ} \beta}^{\beta}, f f^{\prime}\right) \geqq\left(g^{\prime}, f^{\prime}\right),
$$

which is a contradiction. This proves the 'only if' part of the theorem. Conversely suppose that either $g \varphi_{\alpha \circ \beta}^{\alpha}<g^{\prime} \varphi_{\alpha \circ \beta}^{\beta}$ or $g \varphi_{\alpha \circ \beta}^{\alpha}=g^{\prime} \varphi_{\alpha \circ \beta}^{\beta}$, $f<f^{\prime}$. Now we suppose that $(g, f)<\left(g^{\prime}, f^{\prime}\right)$ were not true. Then we would have $(g, f) \geqq\left(g^{\prime}, f^{\prime}\right)$. If $(g, f)=\left(g^{\prime}, f^{\prime}\right)$, then $\alpha=\beta, g=g^{\prime}$, $f=f^{\prime}$, and so

$$
g \varphi_{\alpha \circ \beta}^{\alpha}=g=g^{\prime}=g^{\prime} \varphi_{\alpha \circ \beta}^{\beta},
$$

which is a contradiction. If $(g, f)>\left(g^{\prime}, f^{\prime}\right)$, then, by the result proved above, we would have either $g \varphi_{\alpha_{\circ \beta}}^{\alpha}>g^{\prime} \varphi_{\alpha \circ \beta}^{\beta}$ or $g \varphi_{\alpha \circ \beta}^{\alpha}=g^{\prime} \varphi_{\alpha \circ \beta}^{\beta}, f>f^{\prime}$, which is also a contradiction. This completes the proof.

5. In this section, we argue conversely, and we show that the theorems in $\$ 4$ really characterize ordered completely regular semigroups. More precisely

THEOREM 6. Let $E$ be an ordered idempotent semigroup. For each $\alpha$ of the associated semilattice $T_{E}$ of $E$, suppose that there: 
corresponds an ordered group $G(\alpha)$. Moreover, for each pair of elements $\alpha, \beta \in T_{E}$ such that $\alpha \geqq \beta$, suppose that there corresponds $a$ mapping $\varphi_{\beta}^{\alpha}$ of $G(\alpha)$ into $G(\beta)$ which satisfies the following conditions:

(a) $\varphi_{a}^{\alpha}$ is the identity mapping of $G(\alpha)$;

(b) if $\alpha \geqq \beta \geqq \gamma$, then $\varphi_{\beta}^{\alpha} \varphi_{\gamma}^{\beta}=\varphi_{\gamma}^{\alpha}$;

(c) $\varphi_{\beta}^{\alpha}$ is a group-homomorphism of $G(\alpha)$ into $G(\beta)$;

(d) $\varphi_{\beta}^{\alpha}$ is isotone.

For $\alpha \in T_{E}$, we denote by $S(\alpha)$ the set consisting of all pairs $(g, f)$ with $g \in G(\alpha), f \in E(\alpha)$, where $E(\alpha)$ is the D-equivalence class of $E$ which corresponds to $\alpha \in T_{E}$. Moreover we denote by $S$ the set-union of $S(\alpha)$ when $\alpha$ goes through all the elements of $T_{E}$. We define in $S$ the multiplication by:

if $(g, f) \in S(\alpha),\left(g^{\prime}, f^{\prime}\right) \in S(\beta)$, then

$$
(g, f)\left(g^{\prime}, f^{\prime}\right)=\left(\left(g \varphi_{\alpha \circ \beta}^{\alpha}\right)\left(g^{\prime} \varphi_{\alpha \circ \beta}^{\beta}\right), f f^{\prime}\right),
$$

and define the order by:

if $(g, f) \in S(\alpha),\left(g^{\prime}, f^{\prime}\right) \in S(\beta)$, then

$$
\begin{aligned}
& (g, f)<\left(g^{\prime}, f^{\prime}\right) \text { if and only if either } g \varphi_{\alpha \circ \beta}^{\alpha}<g^{\prime} \varphi_{\alpha \circ \beta}^{\beta} \\
& \qquad \text { or } g \varphi_{\alpha \circ \beta}^{\alpha}=g^{\prime} \varphi_{\alpha \circ \beta}^{\beta}, f<f^{\prime} .
\end{aligned}
$$

Then $S$ is an ordered completely regular semigroup.

Proof. We prove the theorem by dividing into several steps.

$1^{\circ}$. Algebraically $S$ is a completely regular semigroup.

In fact, for $(g, f) \in S(\alpha),\left(g^{\prime}, f^{\prime}\right) \in S(\beta),\left(g^{\prime \prime}, f^{\prime \prime}\right) \in S(\gamma)$, we have

$$
\begin{aligned}
& \left((g, f)\left(g^{\prime}, f^{\prime}\right)\right)\left(g^{\prime \prime}, f^{\prime \prime}\right)=\left(\left(g \varphi_{\alpha \circ \beta}^{\alpha}\right)\left(g^{\prime} \varphi_{\alpha \circ \beta}^{\beta}\right), f f^{\prime}\right)\left(g^{\prime \prime}, f^{\prime \prime}\right) \\
& \left.\quad=\left(\left(\left(g \varphi_{\alpha \circ \beta}^{\alpha}\right)\left(g^{\prime} \varphi_{\alpha \circ \beta}^{\beta}\right)\right) \varphi_{\alpha \circ \beta}^{\alpha \circ \beta}\right)\left(g^{\prime \prime} \varphi_{\alpha \circ \beta \circ \gamma}^{\gamma}\right), f f^{\prime} f^{\prime \prime}\right) \\
& \quad=\left(\left(g \varphi_{\alpha \circ \beta \circ \gamma}^{\alpha}\right)\left(g^{\prime} \varphi_{\alpha \circ \beta \circ \gamma}^{\beta}\right)\left(g^{\prime \prime} \varphi_{\alpha \circ \beta}^{\gamma}\right), f f^{\prime} f^{\prime \prime}\right) \text { by (b) and (c). }
\end{aligned}
$$

Similarly

$$
\begin{aligned}
& (g, f)\left(\left(g^{\prime}, f^{\prime}\right)\left(g^{\prime \prime}, f^{\prime \prime}\right)\right) \\
& \quad=\left(\left(g \varphi_{\alpha \circ \beta \circ \gamma}^{\alpha}\right)\left(g^{\prime} \varphi_{\alpha \circ \beta \circ \gamma}^{\beta}\right)\left(g^{\prime \prime} \varphi_{\alpha \circ \beta \circ \gamma}^{\gamma}\right), f f^{\prime} f^{\prime \prime}\right) .
\end{aligned}
$$

Hence $S$ is a semigroup. Moreover, for $(g, f) \in S(\alpha)$, we have

$$
\begin{aligned}
(g, f)\left(g^{-1}, f\right)(g, f) & =\left(\left(g \varphi_{\alpha}^{\alpha}\right)\left(g^{-1} \varphi_{\alpha}^{\alpha}\right)\left(g \varphi_{\alpha}^{\alpha}\right), f\right) \\
& =\left(g g^{-1} g, f\right) \quad \text { by }(\mathrm{a}), \\
& =(g, f),
\end{aligned}
$$

and also

$$
(g, f)\left(g^{-1}, f\right)=\left(e_{\alpha}, f\right)=\left(g^{-1}, f\right)(g, f),
$$


where $e_{\alpha}$ is the identity of the group $G(\alpha)$. This proves $1^{\circ}$.

$2^{\circ}$. $S$ is simply ordered.

In fact, suppose that $(g, f) \in S(\alpha),\left(g^{\prime}, f^{\prime}\right) \in S(\beta),\left(g^{\prime \prime}, f^{\prime \prime}\right) \in S(\gamma)$. It is clear that $(g, f) \nless(g, f)$, and that at least one of the relations $(g, f)<\left(g^{\prime}, f^{\prime}\right),(g, f)=\left(g^{\prime}, f^{\prime}\right),(g, f)>\left(g^{\prime}, f^{\prime}\right)$ holds. Thus it suffices to prove the transitive law. Hence we suppose that

$$
(g, f)<\left(g^{\prime}, f^{\prime}\right) \text { and }\left(g^{\prime}, f^{\prime}\right)<\left(g^{\prime \prime}, f^{\prime \prime}\right) \text {. }
$$

Now, in the associated semilattice $T_{E}$, we have $\alpha \circ \beta \leqq \beta$ and $\beta \circ \gamma \leqq \beta$. Therefore, by Theorem 1, the two elements $\alpha \circ \beta$ and $\beta \circ \gamma$ of $T_{E}$ are comparable. Without loss of generality, we assume that

$$
\alpha \circ \beta \leqq \beta \circ \gamma \text {. }
$$

Then $\alpha \circ \beta \circ \gamma=\alpha \circ \beta$. By (7) we have

$$
\text { either } g \varphi_{\alpha \circ \beta}^{\alpha}<g^{\prime} \varphi_{\alpha \circ \beta}^{\beta} \text { or } g \varphi_{\alpha \circ \beta}^{\alpha}=g^{\prime} \varphi_{\alpha \circ \beta}^{\beta}, f<f^{\prime},
$$

and

$$
\text { either } g^{\prime} \varphi_{\beta \circ \gamma}^{\beta}<g^{\prime \prime} \varphi_{\beta \circ \gamma}^{\gamma} \text { or } g^{\prime} \varphi_{\beta \circ \gamma}^{\beta}=g^{\prime \prime} \varphi_{\beta \circ \gamma}^{\gamma}, f^{\prime}<f^{\prime \prime} \text {. }
$$

Now we consider by dividing into several cases. First we consider the case when $g \varphi_{\alpha_{\circ \beta}}^{\alpha}<g^{\prime} \varphi_{\alpha \circ \beta}^{\beta}$. Then we have

$$
\begin{aligned}
& \left(g \varphi_{\alpha \circ \gamma}^{\alpha}\right) \varphi_{\alpha \circ \gamma \gamma \gamma}^{\alpha \circ \gamma}=g \varphi_{\alpha \circ \beta \gamma \gamma}^{\alpha}=g \varphi_{\alpha \circ \beta}^{\alpha}<g^{\prime} \varphi_{\alpha \circ \beta}^{\beta}=g^{\prime} \varphi_{\alpha \circ \beta \circ \gamma}^{\beta} \\
& \quad=\left(g^{\prime} \varphi_{\beta \circ \gamma}^{\beta}\right) \varphi_{\alpha \circ \beta \circ \gamma}^{\beta \circ \gamma} \leqq\left(g^{\prime \prime} \varphi_{\beta \circ \gamma}^{\gamma}\right) \varphi_{\alpha \circ \beta \circ \gamma}^{\beta \circ \gamma}=g^{\prime \prime} \varphi_{\alpha \circ \beta \circ \gamma}^{\gamma} \\
& \quad=\left(g^{\prime \prime} \varphi_{\alpha \circ \gamma}^{\gamma}\right) \varphi_{\alpha \circ \beta \circ \gamma}^{\alpha \circ \gamma},
\end{aligned}
$$

and so $g \varphi_{\alpha \circ \gamma}^{\alpha}<g^{\prime \prime} \varphi_{\alpha \circ \gamma}^{\gamma}$. Secondly we consider the case when $g \varphi_{\alpha \circ \beta}^{\alpha}=$ $g^{\prime} \varphi_{\alpha \circ \beta}^{\beta}, f<f^{\prime}, g^{\prime} \varphi_{\alpha \circ \beta \circ \gamma}^{\beta}<g^{\prime \prime} \varphi_{\alpha \circ \beta \circ \gamma}^{\gamma}$ (We remark, since $g^{\prime} \varphi_{\beta \circ \gamma}^{\beta} \leqq g^{\prime \prime} \varphi_{\beta \circ \gamma}^{\gamma}$ by (10), we have always $g^{\prime} \varphi_{\alpha \circ \beta \circ \gamma}^{\beta} \leqq g^{\prime \prime} \varphi_{\alpha \circ \beta \circ \gamma}^{\gamma}$.) Then

$$
\begin{aligned}
\left(g \varphi_{\alpha \circ \gamma}^{\alpha}\right) \varphi_{\alpha \circ \beta \gamma \gamma}^{\alpha \circ \gamma} & =g \varphi_{\alpha \circ \beta \circ \gamma}^{\alpha}=g \varphi_{\alpha \circ \beta}^{\alpha}=g^{\prime} \varphi_{\alpha \circ \beta}^{\beta}=g^{\prime} \varphi_{\alpha \circ \beta \circ \gamma}^{\beta}<g^{\prime \prime} \varphi_{\alpha \circ \beta \circ \gamma}^{\gamma} \\
& =\left(g^{\prime \prime} \varphi_{\alpha \circ \gamma}^{\gamma}\right) \varphi_{\alpha \circ \alpha \circ \gamma}^{\alpha \circ \gamma},
\end{aligned}
$$

and so $g \varphi_{\alpha \circ \gamma}^{\alpha}<g^{\prime \prime} \varphi_{\alpha \circ \gamma}^{\gamma}$. Thirdly we consider the case when $g \varphi_{\alpha_{\circ \beta}}^{\alpha}=$ $g^{\prime} \varphi_{\alpha_{\circ \beta} \beta}^{\beta}, f<f^{\prime}, g^{\prime} \varphi_{\alpha \circ \beta \circ \gamma}^{\beta}=g^{\prime \prime} \varphi_{\alpha \circ \beta \circ \gamma}^{\gamma}, g^{\prime} \varphi_{\beta \circ \gamma}^{\beta}<g^{\prime \prime} \varphi_{\beta \circ \gamma}^{\gamma}$. Then $\beta \circ \gamma \neq \alpha \circ \beta \circ \gamma$. Now $\beta \circ \gamma \leqq \gamma, \alpha \circ \gamma \leqq \gamma$ and so, by Theorem 1, $\alpha \circ \gamma \prec \beta \circ \gamma$ and hence $\alpha \circ \gamma=\alpha \circ \beta \circ \gamma$. Therefore

$$
\begin{aligned}
g \varphi_{\alpha \circ \gamma}^{\alpha} & =g \varphi_{\alpha \circ \beta}^{\alpha}=g \varphi_{\alpha \circ \beta}^{\alpha}=g^{\prime} \varphi_{\alpha \circ \beta}^{\beta}=g^{\prime} \varphi_{\alpha \circ \beta \circ \gamma}^{\beta} \\
& =g^{\prime \prime} \varphi_{\alpha \circ \beta \circ \gamma}^{\gamma}=g^{\prime \prime} \varphi_{\alpha \circ \gamma}^{\gamma} .
\end{aligned}
$$

Moreover we have $f<f^{\prime \prime}$. In fact, otherwise, we would have $f^{\prime \prime} \leqq$ $f<f^{\prime}$. Then, by Lemma 10 , we would have $\alpha \geqq \beta \circ \gamma$, since $f \in E(\alpha)$, 
$f^{\prime} \in E(\beta), f^{\prime \prime} \in E(\gamma)$. Hence we would have $\alpha \circ \beta \circ \gamma=\beta \circ \gamma$, which is a contradiction. Fourthly we consider the case when $g \varphi_{\alpha \circ \beta}^{\alpha}=g^{\prime} \varphi_{\alpha \circ \beta}^{\beta}$, $f<f^{\prime}, g^{\prime} \varphi_{\beta \circ \gamma}^{\beta}=g^{\prime \prime} \varphi_{\beta \circ \gamma}^{\gamma}, f^{\prime}<f^{\prime \prime}$. Then we have $f<f^{\prime}<f^{\prime \prime}$. Moreover, by Lemma 10, we have $\beta \geqq \alpha \circ \gamma$, and so $\alpha \circ \beta \circ \gamma=\alpha \circ \gamma$. Hence

$$
g \varphi_{\alpha \circ \gamma}^{\alpha}=g \varphi_{\alpha \circ \beta \circ \gamma}^{\alpha}=g^{\prime} \varphi_{\alpha \circ \beta \circ \gamma}^{\beta}=g^{\prime \prime} \varphi_{\alpha \circ \beta \circ \gamma}^{\gamma}=g^{\prime \prime} \varphi_{\alpha \circ \gamma}^{\gamma} .
$$

Thus, in all cases, we obtain $(g, f)<\left(g^{\prime \prime}, f^{\prime \prime}\right)$.

$3^{\circ}$. $S$ is an ordered completely regular semigroup.

By $1^{\circ}$ and $2^{\circ}$, it suffices to show that $S$ satisfies the monotone condition:

for $(g, f) \in S(\alpha), \quad\left(g^{\prime}, f^{\prime}\right) \in S(\beta), \quad\left(g^{\prime \prime}, f^{\prime \prime}\right) \in S(\gamma), \quad$ the relation $(g, f)<\left(g^{\prime}, f^{\prime}\right)$ implies $(g, f)\left(g^{\prime \prime}, f^{\prime \prime}\right) \leqq\left(g^{\prime}, f^{\prime}\right)\left(g^{\prime \prime}, f^{\prime \prime}\right)$ and $\left(g^{\prime \prime}, f^{\prime \prime}\right)(g, f) \leqq$ $\left(g^{\prime \prime}, f^{\prime \prime}\right)\left(g^{\prime}, f^{\prime}\right)$.

Here we prove only that $(g, f)\left(g^{\prime \prime}, f^{\prime \prime}\right) \leqq\left(g^{\prime}, f^{\prime}\right)\left(g^{\prime \prime}, f^{\prime \prime}\right)$. By the assumption that $(g, f)<\left(g^{\prime}, f^{\prime}\right)$, we have

$$
\text { either } g \varphi_{\alpha \circ \beta}^{\alpha}<g^{\prime} \varphi_{\alpha \circ \beta}^{\beta} \quad \text { or } \quad g \varphi_{\alpha \circ \beta}^{\alpha}=g^{\prime} \varphi_{\alpha \circ \beta}^{\beta}, f<f^{\prime} \text {. }
$$

We consider by dividing into several cases. First we consider the case when $g \varphi_{\alpha \circ \beta}^{\alpha}<g^{\prime} \varphi_{\alpha \circ \beta}^{\beta}, g \varphi_{\alpha \circ \beta \bigcirc \gamma}^{\alpha}<g^{\prime} \varphi_{\alpha \circ \beta \circ \gamma}^{\beta}$. Then

$$
\begin{aligned}
\left(\left(g \varphi_{\alpha \circ \gamma}^{x}\right)\left(g^{\prime \prime} \varphi_{\alpha \circ \gamma}^{\gamma}\right)\right) \varphi_{\alpha \circ \beta \gamma \gamma}^{x \circ \gamma} & =g \varphi_{\alpha \circ \beta \circ \gamma}^{\alpha} g^{\prime \prime} \varphi_{\alpha \circ \beta \circ \gamma}^{\gamma}<g^{\prime} \varphi_{\alpha \circ \beta \circ \gamma}^{\beta} g^{\prime \prime} \varphi_{\alpha \circ \beta \circ \gamma}^{\gamma} \\
& =\left(\left(g^{\prime} \varphi_{\beta \circ \gamma}^{\beta}\right)\left(g^{\prime \prime} \varphi_{\beta \circ \gamma}^{\gamma}\right)\right) \varphi_{\alpha \circ \beta \circ \gamma}^{\beta \circ \gamma} .
\end{aligned}
$$

(We remark that, in an ordered group, $g<g^{\prime}$ implies $g g^{\prime \prime}<g^{\prime} g^{\prime \prime}$.) Thus $(g, f)\left(g^{\prime \prime}, f^{\prime \prime}\right)<\left(g^{\prime}, f^{\prime}\right)\left(g^{\prime \prime}, f^{\prime \prime}\right)$. Secondly we consider the case when $g \varphi_{\alpha \circ \beta}^{\alpha}<g^{\prime} \varphi_{\alpha \circ \beta}^{\beta}, g \varphi_{\alpha \circ \beta \circ \gamma}^{x}=g^{\prime} \varphi_{\alpha \circ \beta \circ \gamma}^{\beta}$. Then we have

$$
\begin{aligned}
& \left(\left(g \varphi_{\alpha \circ \gamma}^{\alpha}\right)\left(g^{\prime \prime} \varphi_{\alpha \circ \gamma}^{\gamma}\right)\right) \varphi_{\alpha \circ \beta \circ \gamma}^{\alpha \circ \gamma}=g \varphi_{\alpha \circ \beta \circ \gamma}^{\alpha} g^{\prime \prime} \varphi_{\alpha \circ \beta \circ \gamma}^{\gamma} \\
& \quad=g^{\prime} \varphi_{\alpha \circ \beta \circ \gamma}^{\beta} g^{\prime \prime} \varphi_{\alpha \circ \beta \circ \gamma}^{\gamma}=\left(\left(g^{\prime} \varphi_{\beta \circ \gamma}^{\beta}\right)\left(g^{\prime \prime} \varphi_{\beta \circ \gamma}^{\gamma}\right)\right) \varphi_{\alpha \circ \beta \circ \gamma}^{\beta \circ \gamma} .
\end{aligned}
$$

Moreover $\alpha \circ \beta \neq \alpha \circ \beta \circ \gamma$. Now $\alpha \circ \gamma$ and $\alpha \circ \beta$ are comparable, and so $\alpha \circ \gamma=\alpha \circ \beta \circ \gamma$. Similarly $\beta \circ \gamma=\alpha \circ \beta \circ \gamma$. Since $\alpha \circ \beta \neq \alpha \circ \beta \circ \gamma$, by Lemma 10, neither $f \leqq f^{\prime \prime} \leqq f^{\prime}$ nor $f^{\prime} \leqq f^{\prime \prime} \leqq f$ holds. First we suppose that $f \leqq f^{\prime \prime}$. Then necessarily we have $f^{\prime}<f^{\prime \prime}$. Hence, if $E(\alpha \circ \beta \circ \gamma)$ is $R$-typed, then, by Lemma 11,

$$
\begin{aligned}
f f^{\prime \prime} & =\max \left\{y ; y \in E(\alpha \circ \gamma)=E(\alpha \circ \beta \circ \gamma)=E(\beta \circ \gamma), y \leqq f^{\prime \prime}\right\} \\
& =f^{\prime} f^{\prime \prime} .
\end{aligned}
$$

Now we suppose that $E(\alpha \circ \beta \circ \gamma)$ is $L$-typed. We remark that there is no element of $E(\alpha \circ \beta \circ \gamma)$ between $f$ and $f^{\prime}$. In fact, if $h \in E(\delta)$ is an element of $E$ which lies between $f$ and $f^{\prime}$, then, by Lemma 10 ,

$$
\delta \geqq \alpha \circ \beta>\alpha \circ \beta \circ \gamma \text {. }
$$


Hence, by Lemma 11,

$$
\begin{aligned}
f f^{\prime \prime} & =\min \{y: y \in E(\alpha \circ \gamma)=E(\alpha \circ \beta \circ \gamma), y \geqq f\} \\
& =\min \left\{y ; y \in E(\beta \circ \gamma)=E(\alpha \circ \beta \circ \gamma), y \geqq f^{\prime}\right\}=f^{\prime} f^{\prime \prime} .
\end{aligned}
$$

In a similar way, we can prove that $f f^{\prime \prime}=f^{\prime} f^{\prime \prime}$ in the case when $f^{\prime \prime} \leqq f$. Thus we have $(g, f)\left(g^{\prime \prime}, f^{\prime \prime}\right)=\left(g^{\prime}, f^{\prime}\right)\left(g^{\prime \prime}, f^{\prime \prime}\right)$. Thirdly we consider the case when $g \varphi_{\alpha \circ \beta}^{\alpha}=g^{\prime} \varphi_{\alpha \circ \beta}^{\beta}, f<f^{\prime}$. Then, just as in the preceding case, we have

$$
\left(\left(g \varphi_{\alpha \circ \gamma}^{\alpha}\right)\left(g^{\prime \prime} \varphi_{\alpha \circ \gamma}^{\gamma}\right)\right) \varphi_{\alpha \circ \beta \circ \gamma}^{\alpha \circ \gamma}=\left(\left(g^{\prime} \varphi_{\beta \circ \gamma}^{\beta}\right)\left(g^{\prime \prime} \varphi_{\beta \circ \gamma}^{\gamma}\right)\right) \varphi_{\alpha \circ \beta \circ \gamma}^{\beta \circ \gamma} \cdot
$$

Moreover we have $f f^{\prime \prime} \leqq f^{\prime} f^{\prime \prime}$. Thus $(g, f)\left(g^{\prime \prime}, f^{\prime \prime}\right) \leqq\left(g^{\prime}, f^{\prime}\right)\left(g^{\prime \prime}, f^{\prime \prime}\right)$. This completes the proof of Theorem 6 .

\section{REFERENCES}

1. A. H. Clifford, Semigroups admitting relative inverses, Ann. of Math., 42 (1941), 1037-1049.

2. — Bands of semigroups, Proc. Amer. Math. Soc., 5 (1954), 499-504.

3. P. M. Cohn, Embeddings in semigroups with one-sided division, J. London Math. Soc. 31 (1956), 169-181.

4. P. H. H. Fantham, On the classification of a certain type of semigroup, Proc. London Math. Soc., 10 (1960), 409-427.

5. E. S. Lyapin, Semigroups, (1960) (Russian).

6. D. D. Miller and A. H. Clifford, Regular D-classes in semigroups, Trans. Amer. Math. Soc., 82 (1956), 270-280.

7. T. Saitô, Ordered idempotent semigroups, J. Math. Soc. Japan, 14 (1962), 150-169.

8. - Regular elements in an ordered semigroup, Pacific J. Math., 13 (1963), 263-295.

TOKYO GAKUGEI UNIVERSITY, JAPAN 


\section{PACIFIC JOURNAL OF MATHEMATICS}

\section{EDITORS}

Robert Osserman

Stanford University

Stanford, California

M. G. Arsove

University of Washington

Seattle 5 , Washington
J. DugundjI

University of Southern Califorma: Los Angeles 7, California

Lowell J. Paige

University of California

Los Angeles 24, California

\section{ASSOCIATE EDITORS}

E. F. BECKENBACH

B. H. NeumanN

F. WOLF

K. YosIDA

\section{SUPPORTING INSTITUTIONS}

UNIVERSITY OF BRITISH COLUMBIA

CALIFORNIA INSTITUTE OF TECHNOLOGY

UNIVERSITY OF CALIFORNIA

MONTANA STATE UNIVERSITY

UNIVERSITY OF NEVADA

NEW MEXICO STATE UNIVERSITY

OREGON STATE UNIVERSITY

UNIVERSITY OF OREGON

OSAKA UNIVERSITY

UNIVERSITY OF SOUTHERN CALIFORNIA
STANFORD UNIVERSITY

UNIVERSITY OF TOKYO

UNIVERSITY OF UTAH

WASHINGTON STATE UNIVERSITY

UNIVERSITY OF WASHINGTON

AMERICAN MATHEMATICAL SOCIETY CALIFORNIA RESEARCH CORPORATION SPACE TECHNOLOGY LABORATORIES NAVAL ORDNANCE TEST STATION 


\section{Pacific Journal of Mathematics}

\section{Vol. 14, No. 1 \\ May, 1964}

Richard Arens, Normal form for a Pfaffian .........................

Charles Vernon Coffman, Non-linear differential equations on cones in Banach

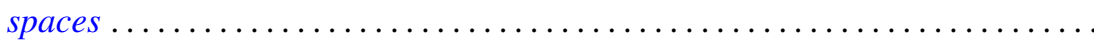

Ralph DeMarr, Order convergence in linear topological spaces ..............

Peter Larkin Duren, On the spectrum of a Toeplitz operator ................

Robert E. Edwards, Endomorphisms of function-spaces which leave stable all

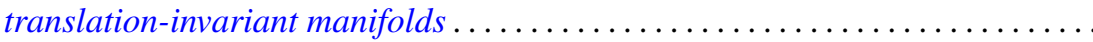

Erik Maurice Ellentuck, Infinite products of isols . . . . . . . . . . . . . . . . 49

William James Firey, Some applications of means of convex bodies . . . . . . . . 53

Haim Gaifman, Concerning measures on Boolean algebras ............. 61

Richard Carl Gilbert, Extremal spectral functions of a symmetric operator. . . . . . 75

Ronald Lewis Graham, On finite sums of reciprocals of distinct nth powers ..... 85

Hwa Suk Hahn, On the relative growth of differences of partition functions ...... 93

Isidore Isaac Hirschman, Jr., Extreme eigen values of Toeplitz forms associated

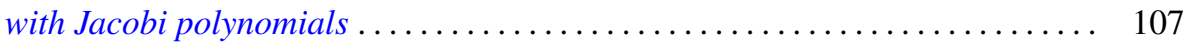

Chen-jung Hsu, Remarks on certain almost product spaces . . . . . . . . . . . 163

George Seth Innis, Jr., Some reproducing kernels for the unit disk . . . . . . . . . 177

Ronald Jacobowitz, Multiplicativity of the local Hilbert symbol . . . . . . . . . . . 187

Paul Joseph Kelly, On some mappings related to graphs ................. 191

William A. Kirk, On curvature of a metric space at a point . . . . . . . . . . . . 195

G. J. Kurowski, On the convergence of semi-discrete analytic functions . . . . . . . 199

Richard George Laatsch, Extensions of subadditive functions . . . . . . . . . . . 209

V. Marić, On some properties of solutions of $\Delta \psi+A\left(r^{2}\right) X \nabla \psi+C\left(r^{2}\right) \psi=0 \ldots 217$

William H. Mills, Polynomials with minimal value sets . . . . . . . . . . . 225

George James Minty, Jr., On the monotonicity of the gradient of a convex

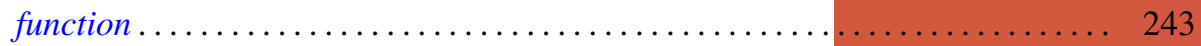

George James Minty, Jr., On the solvability of nonlinear functional equations of 'monotonic' type ................................... 249

J. B. Muskat, On the solvability of $x^{e} \equiv e(\bmod p) \ldots \ldots \ldots \ldots \ldots \ldots \ldots \ldots . \ldots \ldots$

Zeev Nehari, On an inequality of $P . R$. Bessack ................... 261

Raymond Moos Redheffer and Ernst Gabor Straus, Degenerate elliptic

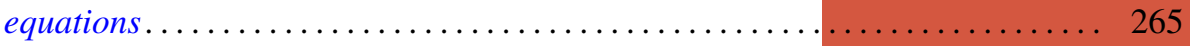

Abraham Robinson, On generalized limits and linear functionals . . . . . . . . . 269

Bernard W. Roos, On a class of singular second order differential equations with a

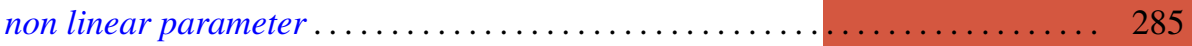

Tôru Saitô, Ordered completely regular semigroups . . . . . . . . . . . . . . . . 295

Edward Silverman, A problem of least area ....................... 309

Robert C. Sine, Spectral decomposition of a class of operators . . . . . . . . . 333

Jonathan Dean Swift, Chains and graphs of Ostrom planes . . . . . . . . . . . 353

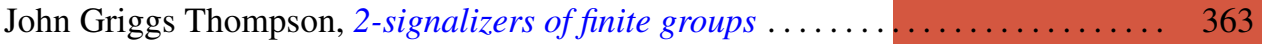

Harold Widom, On the spectrum of a Toeplitz operator . . . . . . . . . . . . . 365 